DOI: $10.2217 / \mathrm{nnm}-2020-0403$

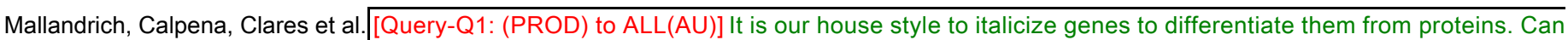
you please check and confirm that any genes in your article are italicized correctly?

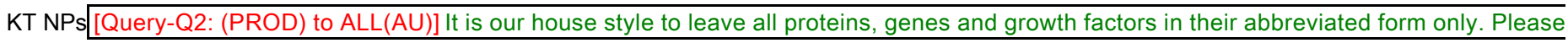

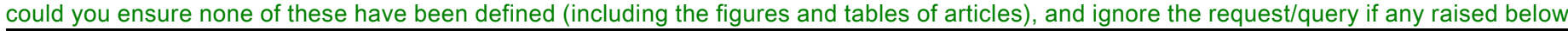

\title{
Nano-engineering of ketorolac tromethamine platforms for ocular treatment of inflammatory disorders
}

Mireia Mallandrich ${ }^{1,2}$, Ana C Calpena ${ }^{1,2}$, Beatriz Clares ${ }^{*}, 2,3$ (D), Alexander Parra ${ }^{1}$, María L García $^{1,2}$, José L. Soriano ${ }^{3}$ \& Francisco Fernández ${ }^{1}$

${ }^{1}$ Department of Pharmacy \& Pharmaceutical Technology \& Physical Chemistry, Faculty of Pharmacy \& Food Sciences, University of Barcelona, 08028 Barcelona, Spain[Query-Q4: (PROD) to $\overline{\mathrm{ALL}(\mathrm{AU})] \text { If you have one and have not already provided it, please provide an ORCID iD for yourself and/or your co-authors. }}$ This should be formatted as follows: https://orcid.org/0000-XXX-XXX-XXXX

${ }^{2}$ Nanoscience \& Nanotechnology Institute (IN2UB), University of Barcelona, 08028 Barcelona, Spain [Query-Q5: Mohit Chopra(APM) to All(AU)] Could you please ensure all affiliations are in full including all details such as department, institution and zip code/postcode?

${ }^{3}$ Department of Pharmacy \& Pharmaceutical Technology, Faculty of Pharmacy, University of Granada, 18071 Granada, Spain

*Author for correspondence: Tel.: +34 958246664; beatrizclares@ugr.es

First draft submitted: 17 October 2020; Accepted for publication: 15 January 2021; Published online: 00000000

\section{Abstract}

Aim: The development and optimization of Ketorolac tromethamine-loaded polylactic-co-glycolic acid nanoparticles (KT-NPs) for the treatment of 
inflammatory processes of the eye. Materials \& methods: KT-NPs were developed by factorial design and characterized by assessing their physicochemical properties. Biopharmaceutical behavior studies, ocular tolerance, anti-inflammatory efficacy and bioavailability tests were performed on pigs. Results: Optimized KT-NPs of $112 \mathrm{~nm}$, narrow distribution with encapsulation efficiency near $100 \%$ were obtained. KT release followed the Weibull model and there was significantly greater retention in the cornea and sclera than in the commercial reference. KT-NPs showed no signs of ocular irritancy and similar anti-inflammatory efficacy to the commercial reference. Conclusion: KT-NPs were a suitable alternative for the treatment of inflammatory disorders of the anterior and posterior segments of the eye as an alternative to conventional topical formulations.

\section{Keywords:}

anti-inflammatory efficacy $\cdot$ factorial design $\cdot$ ketorolac tromethamine $\cdot$ nanoparticles $\cdot$ ocular administration

\section{Funding group}

Ministerio de Ciencia e Innovación http://dx.doi.org/10.13039/501100004837 MAT201459134R

Ocular diseases have a significant effect on the quality of life. Statistical studies demonstrated that around 285 million people living in 39 different countries suffer this kind of deficiency [1]. In many of these common disorders inflammatory processes take place affecting both the anterior and posterior ocular segments [2]. In this context, cataract treatment has been reported to provide the greatest improvement in the quality of life in the community [3]. In fact, cataract surgery is among the most common ophthalmic surgical interventions in developed countries [4].

The use of ophthalmic nonsteroidal anti-inflammatory drugs (NSAIDs) is currently the treatment of choice to prevent and treat this ocular impairment due to their ability to inhibit cyclooxygenase-mediated prostaglandin synthesis [5]. Concretely, diclofenac and ketorolac are the most commonly used NSAIDs [6].

Ketorolac tromethamine (KT) is a NSAID belonging to the pyrrolo-pyrrole group [7]. In ophthalmology, KT is used for the treatment of seasonal allergic conjunctivitis, and postoperative ocular inflammation and pain as an ophthalmic solution [8]. It was approved by international agencies for cataract surgery and currently it is commercially available as eye-drops, Acular $^{\circledR}$, Acular LS $^{\circledR}$ and Acuvail ${ }^{\circledR}$ (Allergan Inc, CA, USA) [9]. However, this conventional dosage form has been widely reported to be inefficient in delivering drugs to posterior segments of the eye due to different ocular barriers, such as the corneal epithelial barrier, as well as other limitations such as the eye's continuous drainage condition [10]. Thus, one of the major objectives in topical 
application to the eye is to achieve an optimal therapeutic level of the drug at the site of action for a prolonged period of time [11].

Nowadays drug inclusion into different delivery systems based on nanostructures, such as polymeric nanoparticles (NPs), generates great clinical expectations. Polymeric NPs are colloidal carrier systems able to improve the ocular bioavailability by the release of drugs in a sustained and prolonged manner at the site of inflammation [12]. Particularly, polylactic-co-glycolic acid (PLGA) is a biodegradable polymer, approved by various national medicines regulatory authorities for ocular application due to its biodegradability and biocompatibility [13].

The main objective of this work was to design and optimize PLGA-NPs for KT encapsulation into a controlled delivery nanoplatform aimed at reaching the biophase at the anterior and posterior segment of the eye. For this task, response surface methodology (RSM) was used to evaluate the best operating conditions. Physicochemical and morphological properties, as well as drug-polymer interaction, in vitro/ex vivo biopharmaceutical behavior were assayed. Finally, in vivo studies comprising tolerance, anti-inflammatory efficacy and bioavailability were also conducted to evidence the effectivity and potential clinical usability of KTPLGA-NPs as a novel therapeutic tool for ocular inflammatory diseases.

\section{Materials \& methods}

\section{Chemicals}

KT, polyvinyl alcohol (PVA) with 90\% hydrolyzation, ethyl acetate and sodium arachidonate acid (SA) were purchased from Sigma-Aldrich (Barcelona, Spain). PLGA (Resomer ${ }^{\circledR}$ RG 503), lactide: glycolide 50:50, Mw $35 \mathrm{KDa}$ were obtained from Boehringer Ingelheim (Ingelheim, Germany). Acular eye drops was purchased from a local pharmacy. All of the chemicals and solvents used were HPLC grade (Merck, Darmstadt, Germany). Purified water was obtained from ultra-pure water Milli-Q plus system (Millipore Iberica S.A.U., Madrid, Spain).

Factorial design

A $2^{3}+$ star central composite rotatable factorial design was addressed to optimize the synthesis of NPs by evaluation of the effects and interactions of three factors or independent variables; drug concentration $\left(\mathrm{X}_{1}\right)$, polymer concentration $\left(\mathrm{X}_{2}\right)$ and $\mathrm{pH}$ of aqueous phase $\left(\mathrm{X}_{3}\right)$, on the dependents variables; mean particle size (Zave), polydispersion index (PI) and encapsulation efficiency (EE). A total of 16 formulations were prepared comprising five different levels for each factor $(-1.68$, lowest; -1 , low; 0 , central; +1 , high and +1.68 , highest) with an additional replicate for the central point. A schematic view of factors and levels is depicted in supplementary data (Supplementary Table 1). Interactions between factors were also studied. Minitab ${ }^{\circledR}$ 
statistical software (Minitab Ltd., PA, USA) was used for this optimization procedure and to calculate the weight of each factor in the polynomic linear equation. An analysis of variance (ANOVA) was performed to elucidate the significance of the effects and interactions between variables. A $p<0.05$ was considered statistically significant.

\section{Preparation of NPs}

NPs were synthetized by the double emulsion-solvent evaporation method, under the optimized conditions determined in the previous section as described elsewhere with modification [13]. Briefly, $500 \mu$ of the inner aqueous phase pH $5.0\left(\mathrm{~W}_{1}\right)$, composed of PVA $1 \%(\mathrm{w} / \mathrm{v})$ and KT $(0.5 \% \mathrm{w} / \mathrm{v})$ solution in phosphate buffer saline (PBS), were added to $2 \mathrm{ml}$ of the organic phase (O), consisting of $100 \mathrm{mg} / \mathrm{ml}$ of PLGA in ethyl acetate solution. This mixture was homogenized using a probe Vibra-Cell ${ }^{\circledR}$ sonicator (Sonics \& Materials, Inc., CT, USA) leading to a primary emulsion (W/O). Then, $4 \mathrm{ml}$ external aqueous phase $\left(\mathrm{W}_{2}\right)$, PVA $2 \%(\mathrm{w} / \mathrm{v})$ solution in PBS, were added dropwise to the primary emulsion, and subsequently it underwent a second sonication process for $90 \mathrm{~s}$, obtaining the double emulsion (W1/O/W2). Finally, $10 \mathrm{ml}$ of PBS pH 7.4 were added to the double emulsion and the organic solvent was evaporated under reduced pressure (Bücchi B-480, Flawil, Switzerland). Unloaded NPs were also elaborated for comparison studies.

\section{Physicochemical \& morphometric characterization}

The mean size (Zave) of the nanoparticle loading ketorolac (Ketorolac tromethamine-loaded PLGA nanoparticle; KT-NP) and PI were determined by dynamic light scattering by using a Zetasizer Nano ZS (Malvern Instruments, Malvern, UK) at $25^{\circ} \mathrm{C}$ with disposable quartz cells.

The optimized KT-NPs were observed by transmission electron microscopy (TEM) with a JEOL 1010 (JEOL Inc., Tokyo, Japan) at $80 \mathrm{Kv}$ and visualized by uranyl acetate solution staining $(1 \%$, w/v). The particle size was measured starting from the TEM image by the image processing program ImageJ (1.53e).

\section{Differential scanning calorimetry}

Samples (KT, PLGA, PVA, KT-NPs and unloaded NPs) underwent DSC analysis using a DSC30 device (Mettler-Toledo, Barcelona, Spain), in the temperature range of $25-200^{\circ} \mathrm{C}\left(10^{\circ} \mathrm{C} / \mathrm{min}\right.$ rate) in a nitrogen atmosphere (50 ml/min). Peak areas were calculated using the Mettler STARe V 9.01 DB software.

\section{Drug loading capacity}

The KT encapsulated in NPs was determined by the indirect method by the measure of the free drug in the dispersion medium. For this task fresh NPs were centrifuged for $15 \mathrm{~min}$ at 14,000 r.p.m. (Sigma 301K, Sigma Laborzentrifugen $\mathrm{GmbH}$, Osterode am Harz, Germany) through Ultracel YM-100 filters (Millipore, MA, USA) to 
separate the nonentrapped KT which was collected in the filtrated solution. EE was determined according to the following equation:[Query-Q6: Mohit Chopra(APM) to All(AU)] could you please provide citation of equation 1 to 4 in text.

$$
E E(\%)=\frac{\text { Total amount of } K T-\text { Free } K T}{\text { Total Amount of } K T} \times 100
$$

The amount of KT was determined by HPLC methodology previously validated in our laboratory [7].

The drug loading (\%DL) of the selected formulation was estimated considering the amount encapsulated in the formulation with respect to the total amount of nanoparticles. For this purpose, NPs were introduced in an oven (to evaporate the solvents) until constant weight. Dried nanoparticles were kept in a desiccator until room temperature was achieved. To obtain loading capacity, the equation suggested by the reviewer was used:

$$
\% D L=\frac{\text { Amount of encapsuled drug }}{\text { Amount of NPS }} \times 100
$$

\section{Stability studies}

The physical stability of the optimized KT-NPs was evaluated by means of static multiple light scattering with a Turbiscan Lab ${ }^{\circledR}$ Expert device (Formulaction, L'Union, France France) using a pulsed near-infrared light source $(\mathrm{Y}=880 \mathrm{~nm})$ for $24 \mathrm{~h}$ at $25^{\circ} \mathrm{C}$. Backscattering (BS) readings were taken just after elaboration and after 7 days storage at room temperature.

\section{Drug release}

Vertical Franz diffusion cells (Vidrafoc, Barcelona, Spain) with a diffusion area of $2.54 \mathrm{~cm}^{2}$ were used to study the KT release from the NPs. A dialysis membrane (MWCO 12,000-14,000 Da., Medicell International Ltd., London, UK) was set between donor and receptor chambers. PBS solution $\mathrm{pH} 7.4$ was used as the receptor medium. The system was kept at $32 \pm 0.5^{\circ} \mathrm{C}$ under magnetic stirring throughout the assay. An amount of $300 \mu \mathrm{l}$ of KT-NPs $0.5 \%(n=6)$ and Acular commercial eye drops $0.5 \%$ as free drug $(n=6)$ were placed into the donor compartment. Sink conditions were maintained throughout the experiment. Aliquots were withdrawn at preestablished times up to $5 \mathrm{~h}$ and then replaced by an equivalent volume of tempered receptor medium.

Experimental data were fitted to five different kinetic mathematical models: zero order, first order, Higuchi, Weibull and Korsmeyer-Peppas by nonlinear least squares regression using the WinNonLin ${ }^{\circledR}$, v. 3.3 software (Pharsight Co., CA, USA). The suitability of model fitting (maximum likelihood) was selected based on the Akaike information criterion. 
Corneal permeation studies

Corneal tissues of New Zealand rabbits (male, weighing 2.5-3.0 kg) for ex vivo permeation studies were obtained from animal facilities of University of Barcelona which were approved by qualified veterinary staff following the ethics committee of animal experimentation of this University. Corneal tissues were mounted on vertical Franz diffusion cells $\left(0.64 \mathrm{~cm}^{2}\right)$ at a constant temperature of $32 \pm 0.5^{\circ} \mathrm{C}$. The osmolarity of samples was rectified using glucose to $250-300 \mathrm{mOs} / \mathrm{kg}$ with a Fiske osmometer. Commercial eye drops (Acular) were used as a reference. One $\mathrm{ml}$ samples were placed in the donor chamber and protected by parafilm ${ }^{\circledR}$ preventing evaporation. Aliquots were collected from the receptor chamber at pre-established times and replaced by the same volume of tempered receptor solution.

The cumulative amount of KT which permeated through the corneal membrane $\left(\mu \mathrm{g} / \mathrm{cm}^{2}\right)$ as a function of time (min) was charted and permeation parameters such as flux (Jss, $\left.\mu g / \mathrm{cm}^{2} \cdot \min \right)$, lag time $\left(T_{L}, \min \right)$, permeability coefficient $(\mathrm{Kp}, \mathrm{cm} / \mathrm{min})$, partition coefficient $\left(\mathrm{P}_{1}\right)$ and diffusion coefficient $\left(\mathrm{P}_{2}\right)$ were calculated.

After permeation study the cornea was dissociated from the sclera ring and cleaned with a $0.05 \%$ sodium lauryl sulfate solution. Then, it was washed with double distilled water, weighed and treated with methanol: water (50:50, v/v) under sonication during 30 minutes using an ultrasound bath for the determination of KT by HPLC. The results are reported as median values (minimum-maximum range) of six and three replicates for the amount of KT permeated and retained, respectively. For comparison results Mann-Whitney test was conducted. p-values $<0.05$ were considered to be significant. The Prism ${ }^{\circledR}$, v. 3.00 software (GraphPad Software Inc., CA, USA) was used for statistical analysis.

In vitro ocular tolerance

The in vitro ocular test was performed using the chorioallantoic membrane (CAM) of embryonated chicken eggs (10 days old). The CAM was exposed to $300 \mu$ l of blank NPs $(n=3)$, KT-NPs $(n=3)$, Acular $(n=3), 0.1 \mathrm{~N}$ $\mathrm{NaOH}$ (positive control, $\mathrm{n}=1$ ), $1 \%$ sodium lauryl sulfate (positive control, $\mathrm{n}=1$ ) and $\mathrm{NaCl}$ (negative control, $\mathrm{n}=1$ ). The ocular irritation index (OII) was calculated with the following equation [10]:

$$
\mathrm{OII}=\frac{(301-\mathrm{h}) \times 5}{300}+\frac{(301-1) \times 7}{300}+\frac{(301-\mathrm{c}) \times 9}{300}
$$

Where, $\mathrm{h}$ is the time (in seconds) of the first appearance of blood hemorrhages, $\mathrm{I}$ is the time (in seconds) of the first appearance of vessel lysis and $c$ is the time (in seconds) of the first appearance of protein coagulation on CAM. 
The comparison between positive and negative controls with the results obtained for the tested formulations were evaluated by three different researchers in a blind test. The evaluations of the researchers were performed separately to avoid possible bias between them.

In vivo studies

In vivo studies were conducted using female Landrace-large white pigs (3-4-month-old). The animals were first acclimatized to room conditions of the Animal Facility at Bellvitge Campus, University of Barcelona, for 1 week before the beginning of the studies. Animal experiments were carried out in strict compliance with the requirements laid down by the association for research in vision and ophthalmology resolution for the use of animals in research. This study was approved by the animal research ethical committee of the University of Barcelona, based on the regulations of the Spanish Government (Royal Decree 53/2013, 1 February, based on European directive 2010/63/UE) with appropriate veterinary supervision.

\section{Ocular tolerance}

The principles of the 3Rs (reduction, refinement and replacement) were carefully followed for in vivo eye irritation. Samples $(50 \mu \mathrm{l})$ of KT-NPs or Acular were instilled on the right eye, using PBS as control on the contralateral eye. Observations to check the appearance of irritation phenomena evaluating the potential damage of conjunctiva, iris and cornea were carried out just after administration and after 15 min.

\section{Anti-inflammatory studies}

Two different experiments were carried out, the prevention of the inflammatory reaction (prophylaxis) and the anti-inflammatory effect itself. Osmolality and the $\mathrm{pH}$ of each formulation, KT-NPs, blank-NPs and Acular eye drops were adjusted before administration. SA was used to provoke the inflammation to the animals, it was prepared at $0.5 \% \mathrm{w} / \mathrm{v}$ dissolved in PBS $\mathrm{pH}$ 7.4. A unique instillation was done in the in vivo experiments: a single dose applying one drop of the formulation (KT-NPs, blank-NPs or Acular) to the eye.

To assess the prevention ability on inflammation of the formulations, one drop of formulation (KT-NPs, blank-NPs or Acular) was administered on the conjunctival sac of the right eyes of pigs. After 30 min, ocular inflammatory was provoked by the instillation of $50 \mu \mathrm{l} \mathrm{SA}$ to the same right eyes. SA was administered to all animal groups including the control group. The left eye was used as a control by the instillation of isotonic saline solution (negative control). Inflammation was evaluated at hourly intervals up to $5 \mathrm{~h}$ after SA administration.

In the second case, the anti-inflammatory efficacy was performed in the same groups of animals after $96 \mathrm{~h}$ of wash-out period. The SA was administrated to the anesthetized animals 20 min later than the formulations, once the inflammatory reaction was evident. KT-NPs and Acular treated groups were also balanced between assays. The inflammation reaction was evaluated after 30, 60, 90, 120 and 150 min of samples administration. 
Ocular bioavailability

After $8 \mathrm{~h}$ of formulation administration, the animals were sacrificed and their eyes were extracted. Vitreous and aqueous humors were surgically extracted from the treated eyes. Samples were treated with solution used as the mobile phase in the HPLC technique to analyze the amount of KT which was retained in different anatomical parts of the eye, such as the sclera, cornea and humors.

\section{Results}

\section{Optimization \& characterization of NPs}

Pure error sum of squares of estimations required a total of 16 experiments as shown in Table 1. [Query-Q7: $\underline{(P R O D)}$ to $A L L(A U)]$ Please check and confirm that any figures/tables that appear in the article have been redrawn correctly, and if not, please describe any corrections that need to be made. Zave of samples ranged between $104.4 \pm 1.69 \mathrm{~nm}$ and $185.5 \pm 1.70 \mathrm{~nm}$. The Zave also showed a narrow distribution with PI values $<0.079 \pm 0.065$. Results of response surface analysis are depicted in Figure 1A and Figure 1B. As can be appreciated, Zave of NPs exhibited minimum values for PLGA concentration rank 4.2-4.5\% (w/v), whereas Zave experiences an increase with KT (Figure 1A). On the other hand, as shown in Figure 1B, EE values of NPs escalated as KT concentration rose up to $0.625 \%(w / v)$. Regarding EE in relation to PLGA concentration, the maximum EE value was observed at $5 \%(w / v)$ which corresponds to the central level of PLGA in the experimental design (Supplementary Table 1). Similarly, the highest EE rates were achieved at $\mathrm{pH}=5$, which is also the central level. [Query-Q8: (PROD) to $\underline{\mathrm{ALL}(\mathrm{AU})] \text { Your figures are currently being redrawn by our graphics department and so they have not been included yet. However, these will be }}$ added before I send you the revised version of the article with your corrections made.

Table 1. Coded and actual values of the three experimental factors: amount of ketorolac tromethamine, amount of nolvlactic-co-alvcolic acid. $\mathrm{nH}$ values of aaueous nhase accordina to the matrix desianed by $2^{3}+$ star central comnosite rotatable factorial desian, and measured responses: average size (Zave), polydispersity index and entrapment efficiency.

\begin{tabular}{|cccccccc|}
\hline \multicolumn{9}{c}{ Coded levels of factors } & \multicolumn{3}{c|}{ Measured responses } \\
\hline & & $\begin{array}{l}\text { KT } \\
(\% \mathrm{w} / \mathrm{v})\end{array}$ & $\begin{array}{l}\mathrm{PLGA} \\
(\% \mathrm{w} / \mathrm{v})\end{array}$ & $\mathrm{pH}$ & $\begin{array}{l}\text { Z-Ave } \\
(\mathrm{nm}) \pm \mathrm{SD}\end{array}$ & $\mathrm{PI} \pm \mathrm{SD}$ & $\begin{array}{l}\text { EE } \\
(\%) \pm \mathrm{SD}\end{array}$ \\
\hline Factorial points & $\mathrm{F} 1$ & 0.25 & 4.5 & 3.5 & $128.0 \pm 1.67$ & $0.046 \pm 0.008$ & $7.79 \pm 1.65$ \\
\hline & $\mathrm{F} 2$ & 1.0 & 4.5 & 3.5 & $107.4 \pm 0.98$ & $0.049 \pm 0.014$ & $35.21 \pm 3.72$ \\
\hline F3 & 0.25 & 5.5 & 3.5 & $131.8 \pm 2.01$ & $0.047 \pm 0.026$ & $14.6 \pm 2.14$ \\
\hline F4 & 1.0 & 5.5 & 3.5 & $115.5 \pm 1.53$ & $0.048 \pm 0.041$ & $45.66 \pm 4.59$ \\
\hline F5 & 0.25 & 4.5 & 6.5 & $122.8 \pm 1.85$ & $0.059 \pm 0.081$ & $19.70 \pm 3.55$ \\
\hline F6 & 1.0 & 4.5 & 6.5 & $104.5 \pm 1.30$ & $0.055 \pm 0.077$ & $42.11 \pm 3.90$ \\
\hline F7 & 0.25 & 5.5 & 6.5 & $164.2 \pm 2.07$ & $0.079 \pm 0.065$ & $85.47 \pm 4.37$ \\
\hline F8 & 1.0 & 5.5 & 6.5 & $138.2 \pm 1.86$ & $0.066 \pm 0.033$ & $88.63 \pm 3.81$
\end{tabular}




\begin{tabular}{|llllllll|}
\hline Axial points & F9 & 1.25 & 5 & 5 & $104.4 \pm 1.69$ & $0.064 \pm 0.039$ & $55.18 \pm 2.64$ \\
\hline & F10 & 0 & 5 & 5 & $110.3 \pm 1.28$ & $0.040 \pm 0.08$ & $0.00 \pm 0.00$ \\
\hline & F11 & 0.625 & 5.8 & 5 & $119.5 \pm 1.09$ & $0.055 \pm 0.013$ & $26.84 \pm 3.80$ \\
\hline & F12 & 0.625 & 4.2 & 5 & $104.8 \pm 1.57$ & $0.045 \pm 0.082$ & $22.84 \pm 2.73$ \\
\hline F13 & 0.625 & 5 & 7.5 & $113.3 \pm 1.22$ & $0.053 \pm 0.039$ & $80.41 \pm 3.57$ \\
\hline & F14 & 0.625 & 5 & 2.5 & $116.7 \pm 1.54$ & $0.045 \pm 0.067$ & $43.64 \pm 2.94$ \\
\hline & F15 & 0.625 & 5 & 5 & $185.5 \pm 1.70$ & $0.040 \pm 0.056$ & $99.72 \pm 2.45$ \\
\hline & F16 & 0.625 & 5 & 5 & $179.0 \pm 1.93$ & $0.046 \pm 0.039$ & $98.12 \pm 2.83$ \\
\hline
\end{tabular}

EE: Encapsulation efficiency; KT: Ketorolac tromethamine; PI: Polydispersity index; PLGA: Polylactic-co-glycolic acid.

Figure 1. Nanoparticles characterization: Surface response chart of: $(A)$ the effect of the interaction between amounts of KT and PLGA on particle size at $\mathrm{pH}=5.0$; (B) the effect of the interaction between amounts of KT and PLGA on entrapment efficiency. (C) Transmission electron microscopy analysis of optimized KT-NPs.

KT-NP: Ketorolac tromethamine-loaded PLGA nanoparticle; PLGA: Polylactic-co-glycolic acid. [Query-Q9: Pratishtha Gupta(CE) to All(AU)] Please provide a general/main title for Figure 1.

Since Zave of all NPs was suitable for ocular administration, the formulation with best EE was selected for further studies. This formulation was F15 (Table 1) with modifications, composed of PLGA in ethyl acetate 5\% $(\mathrm{w} / \mathrm{v})$, aqueous phase at $\mathrm{pH} 5.0$ and $0.5 \% \mathrm{KT}$. These NPs exhibited a Zave $=112 \mathrm{~nm}, \mathrm{PI}=0.043$ and $\mathrm{EE}=99.01 \%$, which corresponded to a drug loading capacity of $18.24 \%$.

Figure 1C shows the TEM image of the final KT-NPs formulation confirming the regular, homogeneous and round shaped systems. The particle size of the nanoparticles on the TEM image is $69.78 \pm 9.73 \mathrm{~nm}$ ( $\mathrm{min}$ $38.33 \mathrm{~nm}$ and max $89.36 \mathrm{~nm}$ ). This particle size is smaller compared with DLS measurements, since DLS measured the hydrodynamic diameter, whereas TEM measured real particle size.

Interaction studies

DSC experiments are usually carried out to evaluate the physical state of the drug and possible interactions between different compounds. Figure 2 shows the DSC thermograms. In the case of PLGA an endothermic peak was registered at $54.78^{\circ} \mathrm{C}$. This value corresponds to the temperature of glass transition (Tg) of this polymer. PVA thermogram showed two peaks at 79.92 and $193.24^{\circ} \mathrm{C}$ corresponding to the melting and decomposition points. Similarly, the KT thermogram also presents two endothermic peaks, one at $159.90^{\circ} \mathrm{C}$ which corresponds to the melting point, and another at $168.13^{\circ} \mathrm{C}$ which corresponds to $\mathrm{KT}$ decomposition. Blank-NPs present an endothermic peak at around 112 and $117^{\circ} \mathrm{C}$. The peak observed in blank NPs denoted the complex interactions of different system compounds. In addition, different events could be overlapped. The onset temperature of blank nanoparticles begins around $100^{\circ} \mathrm{C}$, which could be caused by the evaporation of 
the water present in nanoparticles. After water evaporation, the interactions between PVA and PLGA polymers by $\mathrm{H}$-bounding could be responsible of the second peak observed. The big enthalpy of these peaks indicates the strong interaction between the components. This second peak is less evident in KT-NPs; then, KT could modify the interactions between PVA and PLGA and form a more continuous structure in the thermal events in NPs with a strong interaction between the components, denoted by the big enthalpy of the observed peaks. KT is not present in its initial crystalline form (because the absence of characteristics peaks in the pure compound), whereas is present in amorphous state.

Figure 2. Differential scanning calorimetry thermograms of Ketorolac tromethamine, poly(d,I-lactic-coglycolic) acid, polyvinyl alcohol, blank-nanoparticles and ketorolac tromethamine-loaded polylactic-co-glycolic acid nanoparticles.

\section{Stability studies}

The BS profiles (\%) at room temperature of KT-NPs are depicted in Figure 3. The left side of the curves corresponds to the bottom of the vial, whereas the right side corresponds to the sample behavior on the top. The BS signals show variations around $\pm 1 \%$ which indicated the absence of destabilizing phenomena. Both the volume fraction (migration) and mean size (coalescence) of droplet did not undergo changes in any of the assayed samples.

Figure 3. Backscattering (\%) profiles of samples as a function of sample height (mm). KT-NPs just after elaboration (A) and KT-NPs after a 7 day storage period at room temperature $(B)$.

KT-NP: Ketorolac tromethamine-loaded PLGA nanoparticle; PLGA: Polylactic-co-glycolic acid.

In vitro release study

Figure 4 shows the cumulative KT release pattern from NPs and commercial eye drops. A fast release ( 2 h) was observed in both cases, being slightly faster and higher in the case of Acular eye drops. The model that statistically best explained the process of release of KT from NPs and eye drops was Weibull equation:

$$
\% R_{t} / \% R_{\infty}=1-e^{-\left(\frac{t}{t_{\alpha}}\right) \cdot \beta}
$$

Figure 4. In vitro release profiles of Ketorolac tromethamine from optimized NPs and the commercial 
reference Acular ${ }^{\circledR}$. It is also showed permeation parameters according to the Weibull equation.

In this equation $\% R_{t}$ represents the percentage of the drug released at time $t, \% R_{\infty}$ corresponds to the total percentage of drug released, $\% R_{t} / \% R_{\infty}$ represents the proportion of drug released at time $t$, td is the time necessary for drug to reach $63.2 \%$ release and $\beta$ is the shape parameter. Results of Weibull parameters are presented in Figure 5. As predicted from the release profile, the maximum released KT was statistically higher $(p<0.05)$ for Acular $(76.32 \%)$ versus the NPs $(68.53 \%)$.

Figure 5. Cumulative ex vivo permeation profiles of KT from the KT-NPs and Acular ${ }^{\circledR}$ eye drops after $6 \mathrm{~h}$ through corneal tissue $(A)$ and scleral tissue $(B)$. Each point represents the mean $\pm S D(n=6)$.

KT-NP: Ketorolac tromethamine-loaded PLGA nanoparticle; PLGA: polylactic-co-glycolic acid; SD: Standard deviation. [Query-Q10: Pratishtha Gupta(CE) to All(AU)] Please provide a general/main title for Figure 5.

\section{Ex vivo corneal \& sclera permeation study}

Figure $6 \mathrm{~A}$ and Figure $6 \mathrm{~B}$ show the results of corneal and scleral permeation studies, espectively. KT-NPs and the commercial reference exhibited similar linear slopes corresponding to steady states of fluxes. Calculated permeation parameters are summarized in Table 2. No statistically significant differences were observed between corneal $\mathrm{J}_{\mathrm{ss}}$ values when KT-NPs and the commercial reference were compared. However, significant differences were observed in $T_{L}$ values, being lower for KT-NPs, diffusion related parameter $P_{2}$, which was higher for KT-NPs, and $Q_{\text {ret }}$, which was also greater for KT-NPs. On the other hand, in the case of scleral tissue, statistically significant differences between formulations were observed in all calculated parameters except for partition coefficient $P_{1}$.

Figure 6. Evaluation of the anti-inflammatory efficacy of the formulations tested. Inflammation induction at time $0 \mathrm{~h}(\mathrm{~A})$; control (PBS) after $150 \mathrm{~min}(\mathrm{~B})$; Acular ${ }^{\circledR}$ after $150 \mathrm{~min}$ (C) and KT-NPs after $150 \mathrm{~min}$ (D); Antiinflammatory prevention on pig eyes treated with PBS, KT-NPs $0.5 \%$ and Acular (E).

KT-NP: Ketorolac tromethamine-loaded PLGA nanoparticle; PBS: Phosphate buffer saline; PLGA: Polylactic-coglycolic acid.

Table 2. Median (min-max) transcorneal and transscleral nermeation narameters and retained amounts of ketorolac tromethamine from ketorolac tromethamine-loaded polylactic-co-glycolic acid nanoparticles $0.5 \%$ and the commercial reference Acular $^{\circledR}$ eye drops. 


\begin{tabular}{|c|c|c|c|c|}
\hline Parameter & KT-NPs & Reference & KT-NPs & Reference \\
\hline $\mathrm{Jss}\left(\mu \mathrm{g} / \mathrm{cm}^{2} \mathrm{~min}\right)$ & $\begin{array}{l}1.14 \\
(0.96-1.23)\end{array}$ & $\begin{array}{l}1.03 \\
(0.86-1.11)\end{array}$ & $\begin{array}{l}0.67^{\ddagger} \\
(0.45-0.89)\end{array}$ & $\begin{array}{l}0.33 \\
(0.27-0.39)\end{array}$ \\
\hline$T_{l}(\min )$ & $\begin{array}{l}59.6^{\dagger} \\
(45.6-62.3)\end{array}$ & $\begin{array}{l}67.1 \\
(60.2-78.8)\end{array}$ & $\begin{array}{l}68.0^{\ddagger} \\
(51.4-84.6)\end{array}$ & $\begin{array}{l}126.5 \\
(112.6-140.4)\end{array}$ \\
\hline $\mathrm{P}_{1}(\mathrm{~cm})$ & $\begin{array}{l}0.11243 \\
(0.09715-0.13692)\end{array}$ & $\begin{array}{l}0.12488 \\
(0.10796-0.15209)\end{array}$ & $\begin{array}{l}0.07854 \\
(0.07153-0.08556)\end{array}$ & $\begin{array}{l}0.07777 \\
(0.07221-0.0833)\end{array}$ \\
\hline$P_{2}\left(\min ^{-1}\right)$ & $\begin{array}{l}0.00280^{\dagger} \\
(0.00267-0.00366)\end{array}$ & $\begin{array}{l}0.00249 \\
(0.00211-0.00277)\end{array}$ & $\begin{array}{l}0.00261^{\ddagger} \\
(0.00197-0.00324)\end{array}$ & $\begin{array}{l}0.00133 \\
(0.00119-0.00148)\end{array}$ \\
\hline $\mathrm{Kp}(\mathrm{cm} / \mathrm{min})$ & $\begin{array}{l}0.00036 \\
(0.00030-0.00038)\end{array}$ & $\begin{array}{l}0.00032 \\
(0.00027-0.00035)\end{array}$ & $\begin{array}{l}0.00021^{\ddagger} \\
(0.00014-0.00028)\end{array}$ & $\begin{array}{l}0.00010 \\
(0.00009-0.00012)\end{array}$ \\
\hline Qret $(\mu \mathrm{g} / \mathrm{g})$ & $\begin{array}{l}297.2^{\ddagger} \\
(276.4-311.5)\end{array}$ & $\begin{array}{l}231.7 \\
(144.4-257.4)\end{array}$ & $\begin{array}{l}690.4^{\dagger} \\
(399.2-910.7)\end{array}$ & $\begin{array}{l}396.1 \\
(296.6-408.9)\end{array}$ \\
\hline $\begin{array}{l}\dagger \text { Represents statis } \\
\ddagger \text { Represents statis } \\
\text { KT-NP: Ketorolac tr }\end{array}$ & $\begin{array}{l}\text { significance } p<0 \\
\text { significance } p<0 \\
\text { hamine-loaded } P L\end{array}$ & oparticle; PLGA: & ctic-co-glycolic acid. & \\
\hline
\end{tabular}

In vitro tolerance test

After 5 min of administration and the observations of CAM no hemorrhage, vascular lysis or coagulation phenomena were appreciated for the KT-NPs nor Acular. On the contrary, these phenomena were appreciated in the positive controls $-\mathrm{NaOH}$ and sodium lauryl sulphate- (Supplementary Figure 1). An OII of 0.05 and 0.07 was obtained for KT-NPs and Acular, respectively.

The following classification based on the Oll scores was used: Oll $\leq 0.9$, slightly irritating; $0.9<0$ OII $\leq 4.9$, moderately irritating; $4.9<$ OII $\leq 8.9$, irritating and $8.9<$ Oll $\leq 21$, severely irritating [10]. Thus, based on the Oll score, it could be concluded that formulations did not induce irritation.

In vivo ocular tolerance

After ocular instillation on experimental animals, no irritation phenomena were observed in different ocular structures such as cornea, iris or conjunctiva (Supplementary Figure 2). None of either of the applied formulations (KT-NPs and commercial reference) showed an irritant effect, classifying them as nonirritating.

\section{Anti-inflammatory efficacy}

Figure 6 shows the anti-inflammatory efficacy results. The inflammation induction at time $0 \mathrm{~h}$ (Figure $6 \mathrm{~A}$ ); control eye, depicted in Figure 6B, exhibited a certain degree of redness on the sclera and the conjunctiva at the end of the assay. In contrast, KT-NPs (Figure 6D) and the commercial reference (Figure 6C) showed full recovery after $150 \mathrm{~min}$. Similar anti-inflammatory effects were observed for KT-NPs and the commercial reference. Figure 6E shows images of pigs' eyes used to test the inflammatory prevention. PBS treated eye 
exhibit eye redness. Administration of KT-NPs and Acular showed markedly reduced ocular irritation. No differences were observed between them.

\section{Ocular bioavailability}

The ocular administration of KT-NPs in animals provided $1.56 \pm 0.08 \mu \mathrm{g} / \mathrm{ml}$ of KT in the aqueous humor and $1.55 \pm 0.07 \mu \mathrm{g} / \mathrm{ml}$ in the vitreous humor. In the case of the commercial reference, the amounts of KT found in animals were $1.46 \pm 0.05 \mu \mathrm{g} / \mathrm{ml}$ in the aqueous humor and $1.44 \pm 0.04 \mu \mathrm{g} / \mathrm{ml}$ in the vitreous humor. Statistically significant differences were observed between values of KT from NPs in aqueous humor and KT from the commercial reference in vitreous humor of animals.

\section{Discussion}

Efforts to control inflammation events in the eye are crucial to the maintenance of anatomical integrity and proper visual functionality as well as to prevent or ameliorate ocular pain. Topical administration is the most attractive therapeutic strategy for this task, but this strategy involves limited residence time and the drug passes through the tear film, mucus layer, conjunctiva and cornea [14]. Factorial design is frequently used to plan research into the development of new efficient drug nanoplatforms [15]. The main factors influencing the properties of formulations were selected based on previous experimental activity and specialized bibliographic research.

The selection of PLGA as a polymer for elaboration of NPs aimed at ocular administration was based on the reported properties that PLGA is able to offer [16]. On the other hand, PVA was used as water-soluble synthetic polymer with surfactant properties to produce the NPs and which forms a hydrophilic layer surrounding the NP surface. Through this mechanism PVA gives more stability to this nanoparticulate system [10].

The optimized formulation F15 was modified by changing the KT concentration $(0.5 \% \mathrm{w} / \mathrm{v})$ to match the final concentrations with the commercial reference Acular. The morphometric properties were also recorded by TEM and which confirmed the suitability of the elaboration methods and their use for eye instillation since the small size of KT-NPs might improve their bio-adhesion to biological membranes [11]. Finally, the EE reached 99.01\% and thus KT-NPs can be compared with the commercial reference Acular eye drops in terms of KT amount. Equally DSC results indicated the absence of free crystalline KT in the particle sample. Furthermore, an increment in Tg was observed in KT-NPs regarding blank-NPs and given that KT is the sole different compound, this increment might be due to the interaction of KT with other components of NPs. Optimal stability properties were also demonstrated by the continuous signal of BS with variations close to $1 \%$ even after 7 days of storage. BS profile greater than $10 \%$ is representative of instability [17]. 
To gain knowledge which enabled prediction of the in vivo behavior, the drug release was evaluated [18]. Mathematical models were used to provide valuable information useful to deepen the knowledge and understanding of the mechanisms and the variety of variables involved [19]. The Weibull equation was the model which statistically best adjusted experimental results. The dimensionless shape parameter $\beta$ is the exponent of Equation 5. [Query-Q11: Pratishtha Gupta(CE) to All(AU)] Could you please provide the citation for Equation 5 in the preceding sentence? It can be used to elucidate the type of mechanism driving the diffusion release. For this purpose, $\beta$ values between 0.75 and 1 are reported to be related to a combined Fickian diffusion and swelling controlled release [20]. [Query-Q12: Pratishtha Gupta(CE) to All(AU)]Please check and confirm that the preceding sentence has been edited correctly. The total amount of KT released was higher from eyes drops as is expected since it is a solution. However, KT release velocity expressed by td (time necessary to release the $63.2 \%$ of the applied dose) was higher for NPs which provided a delayed drug release when compared with Acular.

Data from ex vivo permeation studies provide valuable information to help understand how this behavior takes place and provides a preclinical insight allowing optimization of drug delivery systems [21]. Linear relationships between the cumulative amounts of permeated drug versus time were achieved from the $T_{L}$ on, indicating that the cornea and sclera integrities were maintained throughout the experiment and the permeation rates were constant. Transcorneal Jss values were similar between KT-NPs and Acular. However, significant differences were registered in $T_{L}$ through corneal permeation being shorter for KT-NPs and thus could reach the steady state before Acular. Equally, the corneal retained amounts of KT were also higher in the case of NPs compared with Acular, thus it might be expected to exert a more powerful local action. Regarding sclera permeation, KT-NPs showed statistically better results than the commercial reference in Jss, $T_{L}$ and $K T$ retained amounts. KT crossed the sclera tissue before and in a faster manner from NPs than Acular it means that NPs might improve the diffusiveness of KT. The retained amounts of KT in the sclera were also higher in the case of NPs, which might lead to an improved anti-inflammatory action and they might act as a depot to diffuse to vitreous and aqueous humor in the ocular cavity.

According to the obtained results KT-NPs did not produce irritation. Optimized KT-NPs are well suited for use on damaged or inflamed eyes because they are based on biocompatible materials [22].

Two different studies were addressed to evaluating the anti-inflammatory efficacy of the KT-NPs to verify their therapeutic utility for preventing and treating inflammation and optimized KT-NPs exhibited both actions, prevention and treatment of inflammation. In the first case KT-NPs were able to reduce the conjunctival inflammation and iris hyperemia of pig eyes in a significant way, which also contributes to attenuate ocular inflammation which was previously induced by SA instillation. The pharmacologic action of KT was maintained despite being placed in the NP structure. However, the bio-adhesive properties of PLGA confer the colloidal 
system the potential to enhance the residence time in the ocular mucosa and improve the uptake/internalization into the corneal epithelium as showed ex vivo experiments. In the same sense, the anti-inflammatory efficacy (after inflammation induction) of KT-NPs was similar to commercial eye drops Acular. In this regard, components of this commercial reference also included benzalkonium chloride and edetate disodium (EDTA) to enhance drug permeation through the cornea [16]. The use of benzalkonium chloride might produce the disruption of the corneal epithelium $[23,24]$. EDTA might also augment the drug permeability across the cornea. This enhancement is performed by the elimination of extracellular calcium ions, as EDTA is a calcium chelator and this elimination entails a decrease in the tight junction between epithelial cells [25]. The design of KT-NPs was addressed avoiding using potential aggressive ingredients to avoid physiological interactions with ocular tissues. Hence the ocular treatment of KT-NPs is superior in the alleviation of ocular inflammation than eye drops.

\section{Conclusion}

This study reports the design and optimization of PLGA NPs containing KT elaborated by the double emulsionsolvent evaporation method for use in inflammatory processes of the eye. KT-NPs with optimal Zave, PI values and stability properties were selected. They exhibited ocular biocompatibility with anti-inflammatory potential similar to the commercial reference Acular with the added advantage of providing a higher accumulation into ocular tissues leading to an accumulation or reservoir of KT available to extend the residence time in the ocular biophase and thus it is able to produce a more powerful effect and without containing potential aggressive preservatives. Thus, our optimized KT-NPs can be expected to be great candidates to take into account for further investigation in future clinical stages.

\section{Future perspective}

Inflammatory processes in the eye have a significant impact on the quality of individual patients' lives. They are frequently related to surgical interventions of the eye and the cause of important postoperative visual loss. These surgical procedures performed globally each year are likely to increase as populations age. A clear example of this fact is the increasing demand for cataract surgery. For this reason, the research of therapeutical tools especially designed as ocular anti-inflammatories is nowadays a broad area of great interest. NSAIDs are increasingly employed in ophthalmology to manage inflammation and pain after ocular surgery. They are frequently prescribed as eye drops. The strategy of inclusion of NSAIDs in nanocarriers, such as polymeric nanoparticles is one of the future strategies in this therapeutic area with the intention of achieving bio-adhesive enhancement, sustainable drug release, targeted drug delivery, improved biocompatibility, physical and 
chemical stability, etc. In this context, nanoparticles offer a great opportunity for efficacious ocular drug delivery in such a delicate and relatively closed organ like the eye and a significant challenge for Galenic scientists.

\section{Summary points}

- The use of ophthalmic nonsteroidal anti-inflammatory drugs is currently the treatment of choice to manage inflammation and pain.

- Conventional dosage form of ketorolac tromethamine (KT) for the eye has turned out to be inefficient in delivering $\mathrm{KT}$ to the posterior segment of the eye.

- Polylactic-co-glycolic acid nanoparticles (NPs) have been developed by $2^{3}+$ star central composite rotatable factorial design for ocular delivery of KT.

- The optimized NPs possess optimal physicochemical properties in terms of size, morphology, $\mathrm{pH}$, encapsulation efficiency and stability for their use in the eye.

- $\quad$ The KT released from NPs followed a combined Fickian diffusion and swelling controlled release according to the Weibull equation.

- $\quad$ Retained amounts of KT from NPs in corneal and sclera tissues were higher than those retained in the case of the commercial reference.

- The ocular tolerance of Ketorolac tromethamine-loaded polylactic-co-glycolic acid nanoparticles (KTNPs) was demonstrated both in vitro and in vivo.

- The anti-inflammatory efficacy of the KT-NPs was assayed in vivo and demonstrated to be effective for preventing and treating inflammation to a similar extent to the commercial reference.

- The lack of potential aggressive preservatives in KT-NPs prevents physiological interactions with ocular tissue.

- Promising results encourage further clinical investigation so as to exploit the use of this therapeutic tool for ocular inflammation and pain in humans.

\section{Author contributions}

M Mallandrich, AC Calpena and F Fernández wrote the first draft of the manuscript. B Clares, A Parra, ML Garcia and JL Soriano contributed to the final version of the manuscript. All authors provided critical feedback.

Acknowledgments

The authors would like to thank to Alvaro Gimeno Sanding (PhD) from animal facility of at Universidad of Barcelona for his valuable help in animal studies, as well as Lyda Halbaut Bellowa (PhD) and Nuria Bozal-de Febrer $(\mathrm{PhD})$ from the University of Barcelona for their excellent technical support.

Financial \& competing interests disclosure [Query-Q13: (PROD) to $A L L(A U)]$ Please check that the financial disclosure is correct. If 
Please note that as per PMC's policy, the NIHMS/PMC deposit scheme does not apply if your article is not peer reviewed.

This work was supported by the Spanish Ministry of Science and Innovation (MAT201459134R). The authors have no other relevant affiliations or financial involvement with any organization or entity with a financial interest in or financial conflict with the subject matter or materials discussed in the manuscript apart from those disclosed.

No writing assistance was utilized in the production of this manuscript.

\section{Ethical conduct of research}

Animal study of this work was approved by the animal research ethical committee of the University of Barcelona based on the regulations of the Spanish Government (Royal Decree 53/2013, 1 February, based on European directive 2010/63/UE) with appropriate veterinary supervision. The authors state that they have obtained appropriate institutional review board approval or have followed the principles outlined in the Declaration of Helsinki for all human or animal experimental investigations. [Query-Q14: (PROD) to ALL(AU)] When you are done editing,

please save and then click on sign off in the top right to submit the proofs.

\section{References}

Papers of special note have been highlighted as: • of interest; $\bullet$ of considerable interest

1. Pascolini D, Mariotti SP. Global estimates of visual impairment: 2010. Bri. J. Ophthalmol. 96, 614-618 (2012).

2. Madni A, Rahem MA, Tahir N et al. Non-invasive strategies for targeting the posterior segment of eye. Int. J. Pharm. 530, 326-345 (2017).

3. Katta M, Udani P, Heemraz BS et al. Exploring correlations between change in visual acuity following routine cataract surgery and improvement in quality of life assessed with the Glasgow Benefit Inventory. Eye 32, 1549-1550 (2018).

4. Han JV, Patel DV, Squirrell D, McGhee CN. Cystoid macular oedema following cataract surgery: a review. Clin. Exp. Ophthalmol. 47(3), 346-356 (2019).

- Representative review of about postoperative related cystoid macular edema related to contemporary cataract surgery.

5. Al-Lawati H, Binkhathlan Z, Lavasanifar A. Nanomedicine for the effective and safe delivery of non-steroidal antiinflammatory drugs: a review of preclinical research. Eur. J. Pharm. Biopharm. 142, 179-194 (2019).

6. Fernández-Ferreiro A, Santiago-Varela M, Gil-Martínez M et al. Ocular safety comparison of non-steroidal antiinflammatory eye drops used in pseudophakic cystoid macular edema prevention. Int. J. Pharm. 495(2), 680-691 (2015).

7. Mallandrich M, Fernández-Campos F, Clares B et al. Developing transdermal applications of ketorolac tromethamine entrapped in stimuli sensitive block copolymer hydrogels. Pharm. Res. 34(8), 1728-1740 (2017).

8. Donnenfeld ED, Perry HD, Wittpenn JR et al. Preoperative ketorolac tromethamine $0.4 \%$ in phacoemulsification outcomes: pharmacokinetic-response curve. J. Cataract. Refract. Surg. 32(9), 1474-1482 (2006).

9. Jung JW, Chung BH, Kim EK, Seo KY, Kim TI. The effects of two non-steroidal anti-inflammatory drugs, bromfenac $0.1 \%$ and ketorolac $0.45 \%$, on cataract surgery. Yonsei Med. J. 56(6), 1671-1677 (2015).

10. Parra A, Mallandrich M, Clares B et al. Design and elaboration of freeze-dried PLGA nanoparticles for the transcorneal permeation of carprofen: ocular anti-inflammatory applications. Colloids Surf. B Biointerfaces 136, 935943 (2015).

11. Abrego G, Alvarado $\mathrm{H}$, Souto EB et al. Biopharmaceutical profile of pranoprofen-loaded PLGA nanoparticles containing hydrogels for ocular administration. Eur. J. Pharm. Biopharm. 95(Pt B), 261-270 (2015).

-• Excellent contribution dealing with the development of nanoparticles loading nonsteroidal anti-inflammatory drug for ocular inflammatory disorders. 
12. Morsi N, Ghorab D, Refai H, Teba H. Ketoroloac tromethamine loaded nanodispersion incorporated into thermosensitive in situ gel for prolonged ocular delivery. Int. J. Pharm. 506(1-2), 57-67 (2016).

13. Pretel E, Arias JL, Cabeza L et al. Development of biomedical 5-fluorouracil nanoplatforms for colon cancer chemotherapy: influence of process and formulation parameters. Int. J. Pharm. 530(1-2), 155-164 (2017).

14. Nayak K, Misra M. A review on recent drug delivery systems for posterior segment of eye. Biomed. Pharmacother. 107, 1564-1582 (2018).

15. Rincón M, Calpena AC, Clares B et al. Skin-controlled release lipid nanosystems of pranoprofen for the treatment of local inflammation and pain. Nanomedicine (Lond.) 13(19), 2397-2413 (2018).

16. Cañadas $\mathrm{C}$, Alvarado $\mathrm{H}$, Calpena $\mathrm{AC}$ et al. In vitroex vivo and in vivo characterization of PLGA nanoparticles loading pranoprofen for ocular administration. Int. J. Pharm. 511(2), 719-727 (2016).

17. Soriano-Ruiz JL, Calpena-Capmany AC, Cañadas-Enrich C et al. Biopharmaceutical profile of a clotrimazole nanoemulsion: evaluation on skin and mucosae as anticandidal agent. Int. J. Pharm. 554, 105-115 (2019).

18. Sosa L, Clares B, Alvarado $\mathrm{HL}$ et al. Amphotericin B releasing topical nanoemulsion for the treatment of candidiasis and aspergillosis. Nanomedicine 13(7), 2303-2312 (2017).

19. Sierra AF, Ramírez ML, Campmany AC, Martínez AR, Naveros BC. In vivo and in vitro evaluation of the use of a newly developed melatonin loaded emulsion combined with UV filters as a protective agent against skin irradiation. J. Dermatol. Sci. 69(3), 202-214 (2013).

20. Papadopoulou V, Kosmidis K, Vlachou M, Macheras P. On the use of the Weibull function for the discernment of drug release mechanisms. Int. J. Pharm. 309, 44-50 (2006).

21. Parra A, Clares B, Rosselló $A$ et al. Ex vivo permeation of carprofen from nanoparticles: a comprehensive study through human, porcine and bovine skin as an anti-inflammatory agent. Int. J. Pharm. 501(1-2), 10-17 (2016).

22. Gupta H, Aqil M, Khar RK et al. Sparfloxacinloaded PLGA nanoparticles for sustained ocular drug delivery. Nanomedicine 6, 324-333 (2010).

23. Baudouin C, Labbé A, Liang H, Pauly A, Brignole-Baudouin F. Preservatives in eyedrops: the good, the bad and the ugly. Prog. Retin. Eye Res. 29(4), 312-334 (2010).

24. Chen W, Li Z, Hu J et al. Corneal alternations induced by topical application of benzalkonium chloride in rabbit. PLoS ONE 6(10), e26103 (2011).

25. Furrer P, Mayer JM, Gurny R. Ocular tolerance of preservatives and alternatives. Eur. J. Pharm. Biopharm. 53(3), 263-280 (2002). 